\title{
THE EXPERIMENTAL RESPONSE OF AN IMPACTING PENDULUM SYSTEM
}

\author{
Douglas B. Moore and Steven W. Shaw \\ Department of Mechanical Engineering, Michigan State University, East Lansing, MI 48824, \\ U.S.A.
}

\begin{abstract}
This paper presents experimental results obtained from a harmonically excited pendulum system. The pendulum has rigid barriers which limit the amplitude variation from its central position. It is considered in both the normal (downward) position and in the upright (inverted) position. The overall dynamics of the pendulum include impacts with the rigid constraints, and the system response to sinusoidal excitation includes non-impacting motions, stable subharmonics, and chaotic motions. These were experimentally found to occur in the parameter regions predicted by previous analytical work. This system represents an example of a deceptively simple device which can undergo extremely complicated dynamics. For example, the inverted pendulum was found to have 10 distinct possible steady-state responses at a fixed driving amplitude and frequency, each of which was obtained simply by changing the initial conditions. In addition, the normal pendulum was found to be capable of having impacting steady-state dynamics which coexist with the non-impacting steady-state motion predicted from small oscillation theory.
\end{abstract}

\section{INTRODUCTION}

The device considered in this paper falls into the general class of impacting systems which have been studied by several authors including Veluswami and Crossley [1], Veluswami et al. [2], Masri [3], Masri and Caughey [4], Davies [5], Watanabe [6], and others. Studies of impacting systems using bifurcation theory have been made by Shaw $[7,8]$, Shaw and Holmes [9], Holmes [10], Thompson [11] and Whitson [12]. The predictions and analysis used in this experiment are from Shaw and Rand [13] for the inverted pendulum and Sharif-Bakhtiar and Shaw [14] and Shaw [7,8] for the normal pendulum.

Previous experimental work in non-linear systems has focused on showing the existence of chaos in a particular system or on subharmonic responses; coexistence of several motions is not usually considered. Studies where mechanical experiments were combined with theory include Moon and coworkers [15-17], Shaw [18] and Leven and coworkers [19,20]. In this work, the theoretical predictions and computer simulations from $[7,8,13]$ are compared with a physical experiment.

The system considered is a single degree of freedom impacting pendulum (see Fig. 1; the bar is assumed to be rigid so that the model has a single degree of freedom). The non-linear nature of the problem comes from the impacts at the constraints, which are assumed to be close enough to the pendulum's central position to allow a small angle approximation to be used for the free flight dynamics. Additional assumptions are:

(1) that the coefficient of restitution, which dictates how much energy is lost at impact, is constant (i.e. a simple impact rule);

(2) that impacts are instantaneous; and

(3) that the free flight damping is linear, i.e. viscous, in nature.

The input is provided by a sinusoidal motion of the support structure.

In $[7,8,13]$, predictions were made for the existence and stability of certain subharmonic and chaotic motions of this system. The goal in these experiments was to confirm the dynamic behavior as a function of system parameters as predicted in $[7,8,13]$. The analysis presented in $[7,8,13]$ does not depend on detailed asymptotics or topological ideas; it uses, for the most part, matching and stability methods. In addition, no assumptions on the magnitudes of the driving amplitudes or dissipation were required in the theoretical work.

The paper is arranged as follows: Section 2 provides the equations of motion and a brief description of some of the types of motion which are possible, Section 3 describes the 

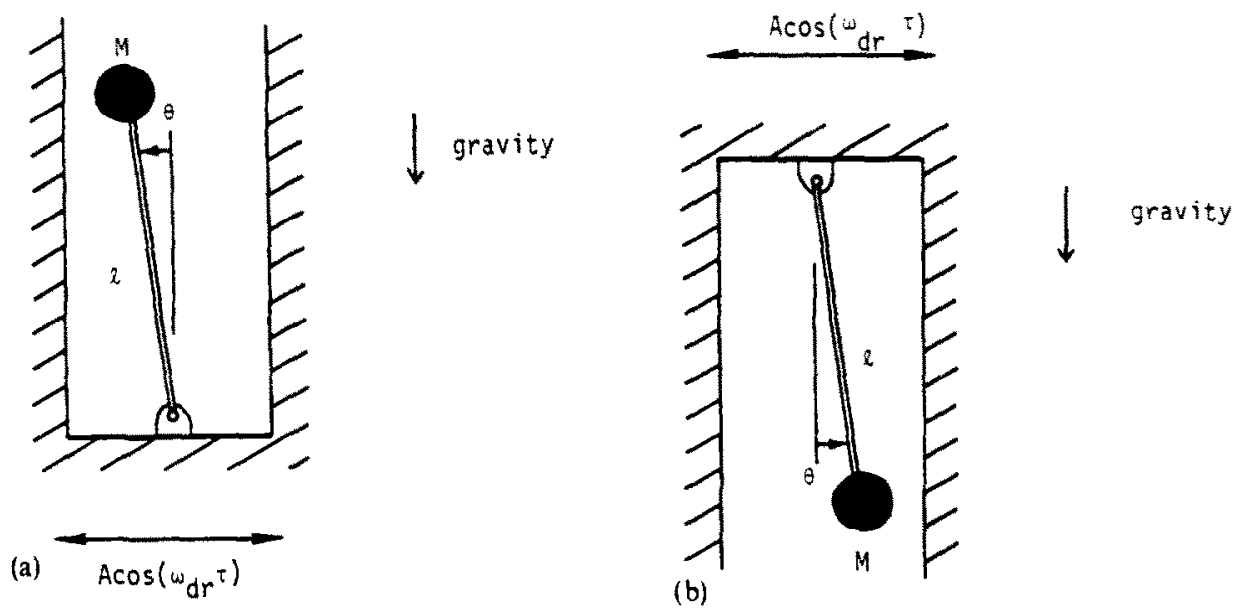

Fig. 1. (a) The inverted pendulum with stops. (b) The normal pendulum with stops.

experimental procedures and apparatus, Section 4 presents the experimental results of the observed motions, with a comparison with the predictions, and the paper closes with a discussion in Section 5.

\section{SUMMARY OF ANALYSIS}

\subsection{Equations of motion}

The equations of motion for the impacting pendulum are as follows:

$$
\begin{aligned}
m l^{2} \theta^{\prime \prime}+b \theta^{\prime} \pm m g l \sin \theta & =l A m \omega_{\mathrm{dr}}^{2} \cos \theta \cos \left(\omega_{\mathrm{dr} r}\right) \quad|\theta|<\theta_{\max } \\
\theta_{+}^{\prime} & =-r \theta_{-}^{\prime}, \quad|\theta|=\theta_{\max },
\end{aligned}
$$

where $m$ and $l$ are the mass and the length of the pendulum, respectively, as shown in Fig. 1(a, b), and the $+($ or - ) sign is used for the regular (or inverted) pendulum. The parameter $A$ represents the amplitude of the support displacement, $b$ is the effective angular viscous damping constant, $g$ is gravitational acceleration, $\omega_{\mathrm{dr}}$ is the frequency of the input motion, $r$ is the coefficient of restitution at impact, and primes denote $d / d \tau$, with $\tau$ representing time. The constraints are placed symmetrically at $\theta= \pm \theta_{\max }$.

The inverted case is considered first. Simplified equations of motion may be obtained by rescaling equation (1). Defining $t=\sqrt{ }(g / l) \tau$ and $\phi=0 / \theta_{\max }$, the non-dimensionalized equations of motion for the system for small angles from the vertical are

$$
\begin{array}{rr}
\ddot{\phi}+2 \alpha \phi-\phi=\beta \cos (\omega t), & |\phi|<1 \\
\dot{\phi}_{+}=-r \dot{\phi}_{-}, & |\phi|=1
\end{array}
$$

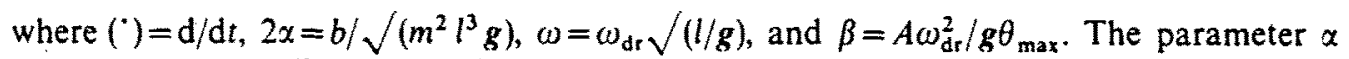
represents the non-dimensional damping ratio, $\beta$ the non-dimensional driving acceleration, $\omega$ the dimensionless driving frequency, and $r$ is the coefficient of restitution. These parameters and the initial conditions will determine the steady-state response of the system.

The equations for the normal (non-inverted) pendulum system are similar to those for the inverted pendulum system. The non-dimensional equations of motion for the normal pendulum are

$$
\begin{aligned}
\ddot{\phi}+2 \alpha \dot{\phi}+\phi & =\beta \cos (\omega t), & & |\phi|<1 \\
\dot{\phi}_{+} & =-r \dot{\phi}_{-}, & & |\phi|=1,
\end{aligned}
$$

where the dimensionless parameters for this case are the same as for the inverted pendulum, with $\theta$ defined as in Fig. 1(b).

The unforced, undamped phase space for the inverted and normal cases are given in Figs 2(a) and (b), respectively. We note that the normal pendulum can undergo linear, non- 

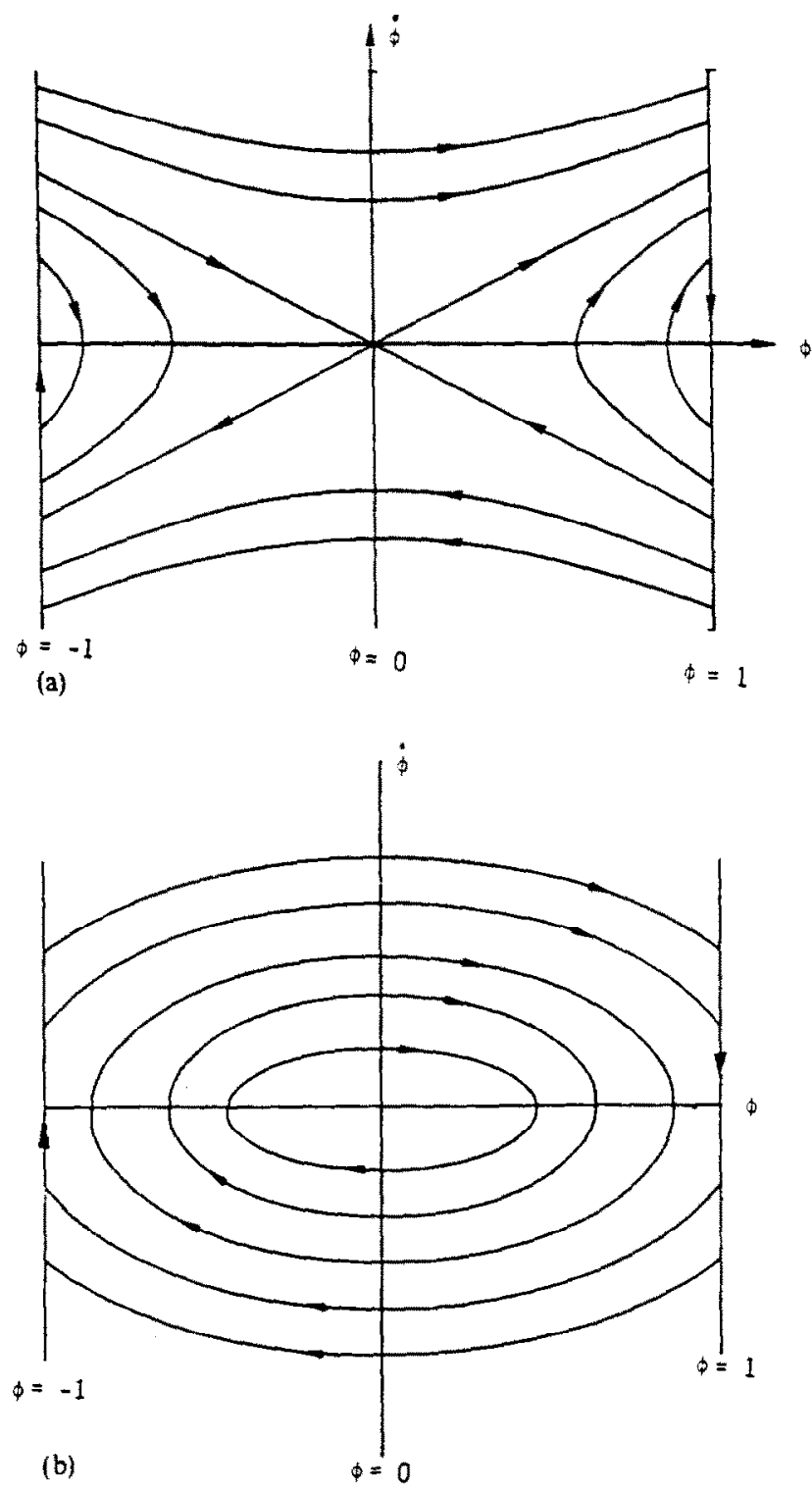

Fig. 2. Undamped, unforced $(x=0, \beta=0, r=1)$ phase portraits. (a) The inverted pendulum. (b) The normal pendulum.

impacting oscillations and that the inverted pendulum has static 'resting' motions possible at either constraint.

\subsection{Existence and bifurcations of periodic motions}

There are two general types of periodic impacting motion, and subclasses of each, which have been predicted for these systems $[7,8,13]$. The two general types will be designated Type I and Type II. A Type I motion is a periodic motion impacting one constraint only, once per period of the motion. A Type II motion is a symmetric periodic motion impacting both constraints. The normal pendulum can undergo motions of Type II along with linear motions which do not impact the constraints. The inverted pendulum can undergo either Type I or Type II motions. It also admits, for $\beta<1$, the 'rest' motion in which the pendulum remains in contact with one wall $[13]$. Both systems can undergo chaotic motions $[7,8,13]$. Other types of periodic motions are possible but have not been dealt with in the analysis of the system; some of these were observed in the experiments and are presented in the following. In the systems studied, three types of local bifurcations were analyzed: saddlenode bifurcations, period doubling bifurcations, and pitchfork bifurcations. A brief discus- 
sion on the periodic motions and the bifurcations is provided here; the reader is referred to the analytical work in $[7,8,13]$ for more details and examples from simulation studies.

A saddle-node bifurcation occurs when a stable motion and an unstable motion converge and annihilate each other at the bifurcation point. Saddle-node bifurcations are a mechanism by which steady-state motions can appear or disappear. Such bifurcations for both Type I motions, impacting one constraint only, and Type II motions, impacting both constraints, are the first to occur as the driving amplitude is increased. As a system parameter is varied, these periodic motions will exist and one will be stable until a secondary bifurcation point; beyond that point the motions will still exist but will be unstable and therefore not experimentally observable. Typically, new stable motions will be born at the secondary bifurcation.

A Pitchfork bifurcation results in the appearance, from a solution branch which represents a symmetric motion, of two new branches which represent an antisymmetric pair of motions. This type of bifurcation is found in systems with symmetry. The two new stable motions are symmetrical about the original motion in the phase space and have the same period as the original motion. Pitchfork bifurcations are the secondary bifurcations for all Type II motions. For parameter values just above the pitchfork bifurcation curves, these antisymmetric motions are stable and can be expected to be observed.

Period doubling bifurcations occur when a motion of period $T(T=2 \pi / \omega)$ becomes unstable and a new motion of period $2 T$ is born. The bifurcation diagram is similar to the pitchfork diagram; the difference is in how the original stable motion loses stability. After a supercritical period doubling the motion essentially flips, or alternates, between the two new stable branches, i.e. the two branches represent the same motion. It should be noted that such a motion will repeat after two impacts, whereas the original Type I motion repeated after each impact. Period doublings occur in the inverted pendulum system when the stable Type I motions become unstable. Period doubling bifurcations often occur in rapid sequence; this is referred to as a period doubling cascade and is a precursor of chaotic motion in the system [21]. The asymmetric descendants of the Type II motions can also undergo period doublings.

\section{EXPERIMENTAL EQUIPMENT AND PROCEDURES}

Design of the experimental apparatus was driven by the desire to match the conditions of the analysis as closely as possible. These included: a single degree of freedom system, i.e. only the rigid body mode active; instantaneous, dissipative impacts at the constraints; linear viscous damping; and a sinusoidal displacement of the support.

The dimensions of the experimental apparatus were as follows. The distance between the constraints, $\simeq 2 l \theta_{\max }$ for $\theta_{\max } \ll 1$, was $6.6 \mathrm{~cm}$. The length of the pendulum, $l$, was $25.3 \mathrm{~cm}$. The pendulum mass, $m$, was approx. $0.05 \mathrm{~kg}$. (The exact values of the end mass and of the pendulum bar are not important as they do not appear in any of the non-dimensional quantities except the damping ratio, and the damping ratio was measured experimentally.) The natural frequency of the system, $\sqrt{ }(\mathrm{g} / \mathrm{l}) / 2 \pi$, was measured to be $0.98 \mathrm{~Hz}$ from small oscillations in the normal position.

The system parameters $x$ and $r$, which remained fixed throughout the experiment, were determined as follows. The coefficient of restitution, $r$, was assumed to be constant. We analyzed the time derivative of an angular displacement signal when impacts were occurring by using the differentiation hardware built into a Hewlett-Packard 5423A structural dynamics analyzer. The coefficient of restitution was assumed to be the absolute value of the ratio of the velocity immediately after impact to the velocity immediately before impact. The measured value was $r=0.95 \pm 0.04$. The uncertainty is caused primarily by the dependence of the coefficient of restitution on the impact velocity. The damping coefficient $x$ was obtained by hanging the pendulum in the normal position, giving the system an initial condition such that did not contact the constraints, and recording the subsequent motion of the pendulum. The logarithmic decrement method was used on the data obtained to determine the damping value, $\alpha=0.03$. A value for the uncertainty of the $x$ value is not known but, as the results depend on $\alpha$ only to the first order, a $100 \%$ error in the damping value, \pm 0.03 , will result in only a $3 \%$ change in the predicted bifurcation curves. The dissipation of energy 
during free-flight is from many sources, including dry friction and aerodynamic damping, and this $\alpha$ value is an equivalent viscous damping parameter.

Minimization of flexural vibrations in the system was a critical design condition. Excitation of these modes at impact is inevitable. The effects of this were minimized by the choice of a bar with high flexural stiffness and relatively large structural damping; this kept flexural displacements small and quickly damped out higher modes. To this end, a tube of graphite composite material was selected for the rod. An analysis of the impact process, and experimental observation of impacts, showed the rod to have insignificant energy in the flexural vibration modes [22]. Also, the energy which is transferred to these modes at impact can be thought of as being lost at impact and lumped in with the coefficient of restitution. This assumption is reasonable, as most of the transient flexural motion which is excited at impacts decays before the next impact occurs. The high stiffness-to-weight ratio of the graphite composite, along with the structural damping of the material, proved to approximate the rigid, massless beam quite well for our purposes. The frequency range for the experiments was under $25 \mathrm{~Hz}$, and the first flexural frequency for this system was estimated to be approx. $300 \mathrm{~Hz}$ (using the method of assumed modes).

A hard rubber ball with nearly elastic rebound characteristics was chosen as the pendulum mass, to provide nearly instantaneous impacts and to minimize the excitation of flexural modes of the bar.

Additional design considerations were to reduce friction in the bearing and to build a rigid structure which would house the system and provide the constraints. Standard ball bearings were chosen to keep the friction small; these were mounted in two pillow blocks to provide uniform support for the pendulum. The frame was constructed out of $5-\mathrm{mm}$ aluminum T-stock; this provided the required rigidity needed for the constraints.

An electrodynamic shaker was used as the driving device. With this shaker, both large forces and clean sinusoidal signals could be applied to the frame without significant feedback from motion and impacts of the pendulum.

The sensing system for the pendulum was built around a Hall effect transducer. A circular permanent magnet was attached to the end of the shaft which rotated through the mounting bearings. The Hall effect sensor was attached to the frame near the magnet to pick up the variations in the magnetic field, and the magnet was oriented such that the pendulum angle was well approximated by a linear function of the output voltage. An accelerometer was mounted to the base of the frame to provide information on the excitation amplitude and frequency.

The signal from the Hall effect sensor and the signal from the accelerometer provided the information required to map out the motions in the $(\beta, \omega)$ parameter space of the system. The signals obtained from the sensors were input into a Hewlett-Packard 5423A structural dynamics analyzer. The Hall effect sensor signal was displayed as a time trace to determine the type of motion present in the system at the particular parameter values. Chaotic motions and the various types of periodic motions were distinguished from one another using visual observation of the motion and its time trace and spectral data obtained using the spectrum analyzer. The acceleration signal from the frame was analyzed in the frequency domain to determine its magnitude at the excitation frequency, $\omega_{\mathrm{dr}}$. The acceleration signal was also analyzed to determine if higher harmonics of the forcing frequency were present. In all cases, additional harmonics in the input acceleration were less than $-20 \mathrm{db}$ in amplitude.

The experimentally observed motions were those which have a robust stability region in parameter space and in initial condition space. At many parameter values there are a large number of stable solutions possible and the initial conditions needed to produce a certain steady state were not known; we simply started the system and watched what developed. In some parameter regions the model predicts periodic motions, typically those of large periods, which have extremely small stability windows in parameter space, and the set of initial conditions which lead to that motion, i.e. the domain of attraction, is very small. If the noise level is such that the associated disturbances are larger than the domain of attraction or the stability window of a given motion, then that motion will not be observed in the experiment. One consequence of this is that there are motions predicted by the theory which can never be observed; these are typically those of very long period which, in any case, may be practically indistinguishable from chaos. 
It should also be pointed out that, due to the rescaling used, the actual driving frequency, $\omega_{\mathrm{dr}}$, appears in both the non-dimensional driving frequency, $\omega$, and in the non-dimensional driving amplitude, $\beta$. The results below are summarized by indicating the various dynamic responses in $(\beta, \omega)$ space. If one carries out a sweep in the driving amplitude, $A$, at a fixed driving frequency, $\omega_{\mathrm{dr}}=$ constant, this corresponds to moving along a vertical line in $(\beta, \omega)$ space. On the other hand, a frequency sweep of $\omega_{\mathrm{dr}}$ at a fixed amplitude, $A=$ constant, corresponds to moving along a parabolic path in $(\beta, \omega)$ space, as it is the quantity $\beta / \omega^{2}$ which remains fixed as $\omega_{\mathrm{dr}}$ is varied and $A$ is held fixed. To hold $\beta$ fixed and vary $\omega$, one would need to vary $\omega_{\mathrm{dr}}$ and simultaneously adjust $A$ such that $A \omega_{\mathrm{dr}}^{2}$ remains constant.

\section{RESULTS}

This section describes the experimentally obtained dynamics of the impacting pendulum system. It contains individual discussions of the inverted pendulum and normal pendulum results, comparing each with the analytical results. The discussion in each case provides a general view of certain scenarios which one may encounter as parameters are varied.

The responses of the pendulum are demonstrated in the following by showing traces of a voltage which is proportional to the pendulum angle vs time. The plots also indicate the excitation signal directly below the pendulum response signal. The vertical scales vary from picture to picture and are not given, as we are primarily interested in different types of qualitative behaviors. In fact, the scales are adjusted such that each signal fills the vertical extent of the plot; this was convenient for determining the qualitative nature of the signals as many of the Type I responses were of very low amplitude.

\begin{tabular}{|c|c|}
\hline Key of $M$ & tions (Type \\
\hline $\begin{array}{l}\text { A }-I, 1,1 \\
B-1,2,1 \\
C-I, 3,1 \\
D-I, 4,1 \\
E-I, 5,1 \\
F-1,2,2 \\
G=1,4,2 \\
H-R e s t \\
P=1,6,2\end{array}$ & 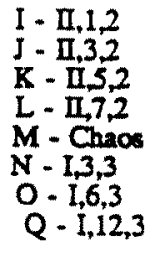 \\
\hline
\end{tabular}

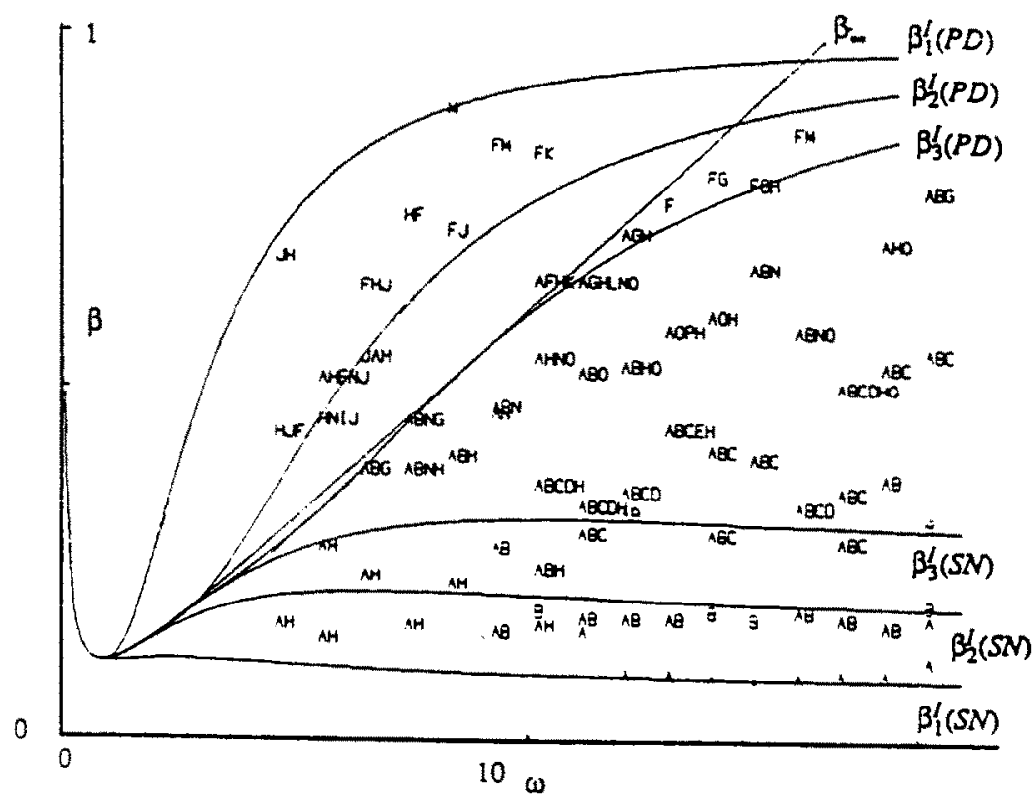

Fig. 3. Experimental results for Type I and related motions, inverted pendulum. Curves are the predicted bifurcation conditions from Shaw and Rand [13]. 


\subsection{Inverted pendulum}

The results of the experiments for the inverted pendulum are given in Figs 3 and 4. These figures show the observed motions vs the predicted bifurcation curves (from Shaw and Rand $[13])$ in the $(\beta, \omega)$ parameter space for Type I and Type II motions, respectively. The letter strings in the figures correspond to various motions which were observed at the designated point in parameter space given by the lower left corner of the first letter. The curves on the figures are the bifurcation conditions from [13], above which the indicated motion exists. For Fig. 3, the $\beta_{n}^{1}(\mathrm{SN})$ curves give the conditions above which we expect to find Type I periodic motions of period $n T$, impacting one wall only. These motions, born in saddle-node bifurcations, will exist and be stable until the corresponding $\beta_{n}^{\prime}(\mathrm{PD})$ curve is reached, at which point the Type I motion loses stability and a new motion of period $2 T$ with two impacts per period is born in a period doubling bifurcation. Therefore, in the region between a $\beta_{n}^{l}(\mathrm{SN})$ curve and a $\beta_{n}^{\mathrm{l}}(\mathrm{PD})$ curve we expect to observe a Type I motion of period $n T$. Just above the $\beta_{n}^{\prime}(\mathrm{PD})$ curves, we expect to observe the associated period $2 T$ motions. Further above the $\beta_{n}^{1}(\mathrm{PD})$ curves it is reasonable to guess that period doubling cascades will occur, leading to chaos.

Data were taken so as to find the lowest $\beta$ values for which certain motions existed over a range of frequencies. For frequencies in the range 10-20 (non-dimensionalized) the agreement with the predicted bounds is good.

Motions corresponding to $\beta_{1}^{\prime}(\mathrm{SN})$ (period $T=2 \pi / \omega_{\mathrm{dr}}$ ) are given by the letter A in Fig. 3 . The $\beta_{2}^{\prime}(\mathrm{SN})$ curve gives the parameter values above which we expect to see Type I, period $2 T$ motions, corresponding to the letter B. Figure 5(a) shows a time trace of such a motion. Again, the agreement between the predicted values and the experimental results in Fig. 4 should be noted. The $\beta_{3}^{\prime}(\mathrm{SN})$ curve gives the parameter values above which we expect

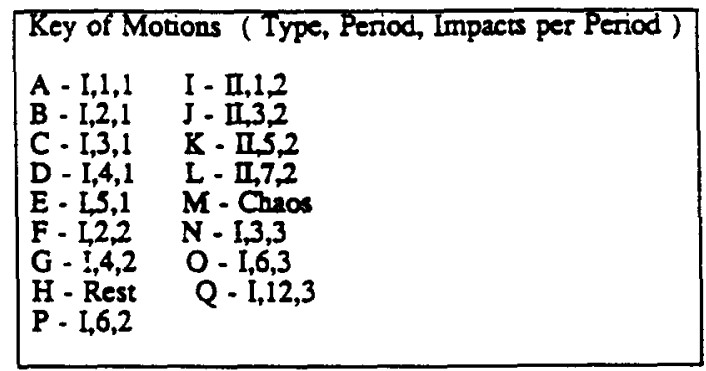

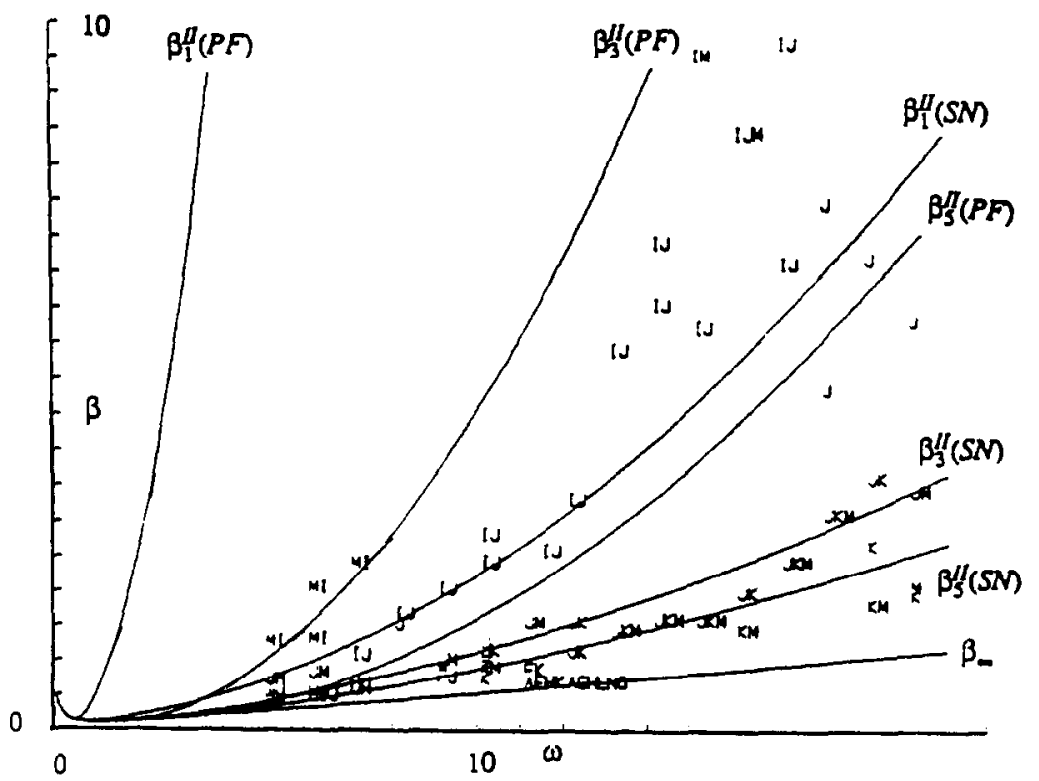

Fig. 4. Experimental results for Type II and related motion, inverted pendulum. Curves are the predicted bifurcation conditions from [13]. 
motions corresponding to $C$ to exist; Fig. $5(\mathrm{~b})$ shows a motion corresponding to $C$. It is noteworthy that in the region above the $\beta_{3}^{\prime}(\mathrm{SN})$ curve and below the $\beta_{3}^{\perp}(\mathrm{PD})$ curve $\mathrm{A}, \mathrm{B}$ and $C$ motions exist and are stable. Figure 5(c) depicts a Type I, period $4 T$ motion corresponding to the letter $\mathrm{D}$. The letter $\mathrm{E}$ corresponds to a Type I motion of period $5 T$ with one impact per period.

The $\beta_{1}^{\prime}$ (PD) curve gives the parameter values for which we expect the A motions to lose stability and motions corresponding to the letter $F$ to arise. The letter $G$ corresponds to motions which arise from period doublings of Type I, period $2 T$ motions; an example of such a period $4 T$ motion is shown in Fig. 6 . The agreement between the experimental results and the predictions is not as good as for the $\beta_{n}^{\prime}(\mathrm{SN})$ motions but the general trend is as expected.
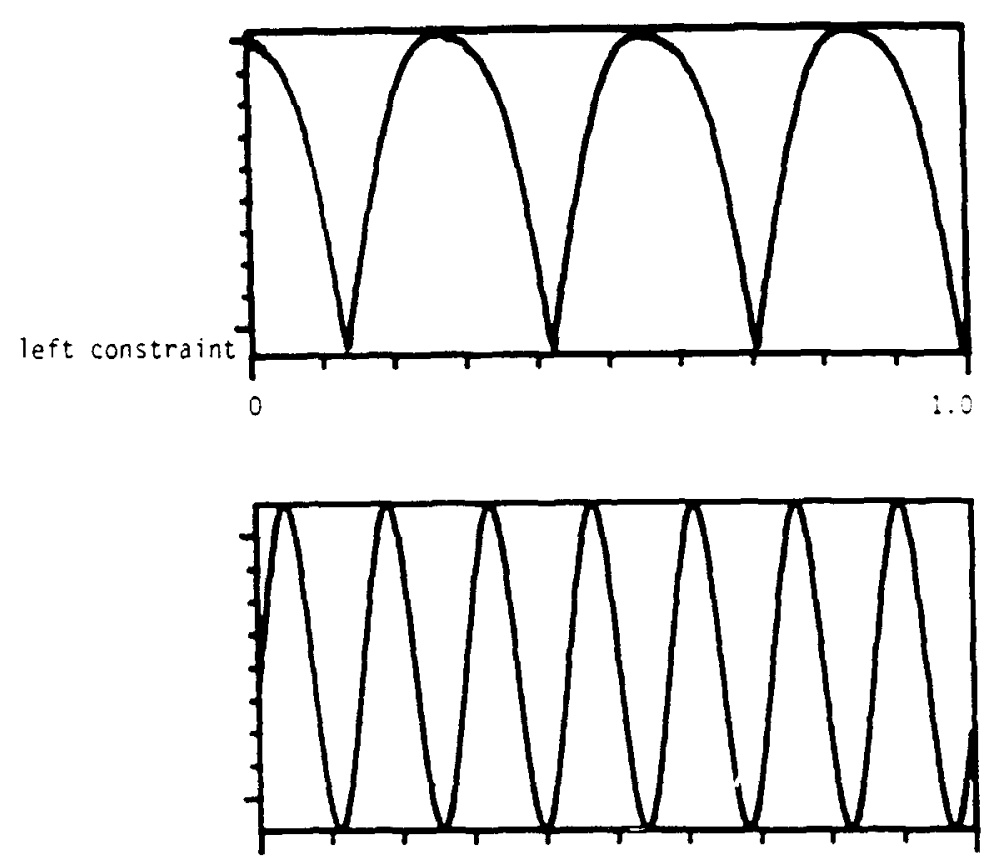

(a)
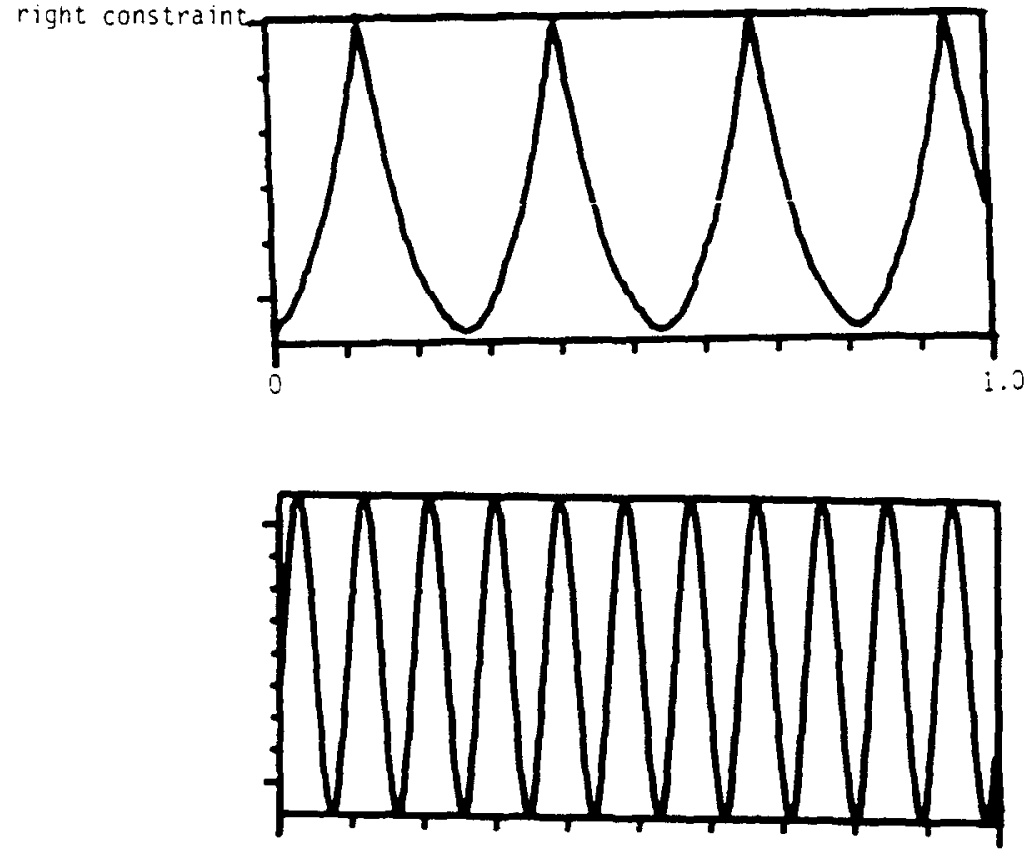

(b) 0 


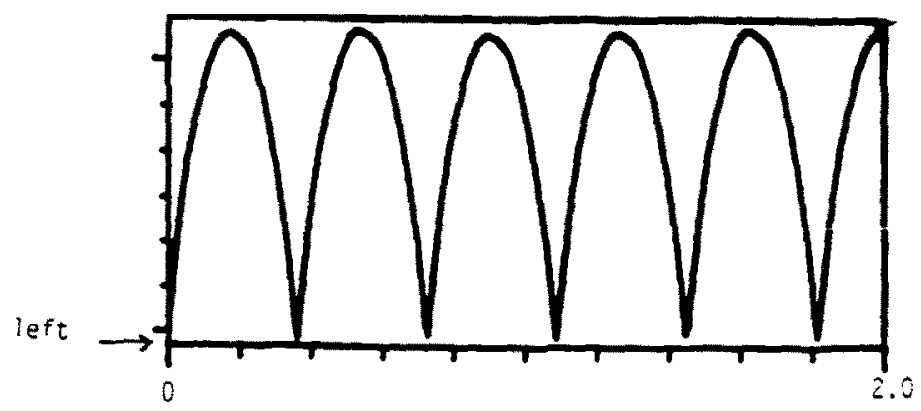

(c)

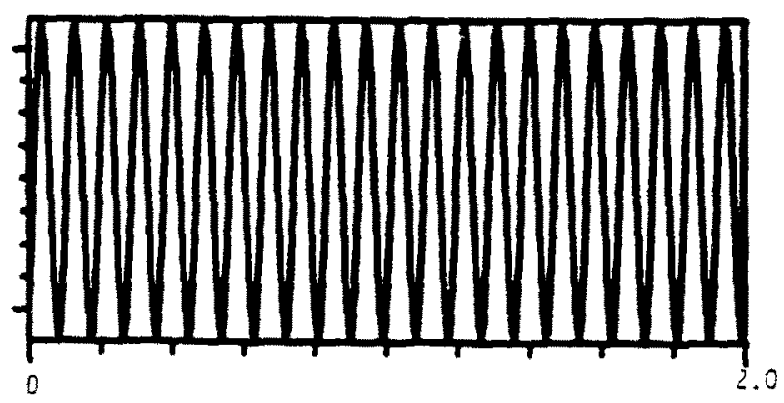

Fig. 5. Type I motions; inverted pendulum. (a) Period $2 T$; letter B in Fig. $5 ;(\beta, \omega)=(0.38,7.09)$. (b) Period $3 T$; letter C in Fig. $5 ;(\beta, \omega)=(0.36,11.14)$. (c) Period $4 T$; letter D in Fig. $S ;(\beta, \omega)=(0.36$, 11.14).
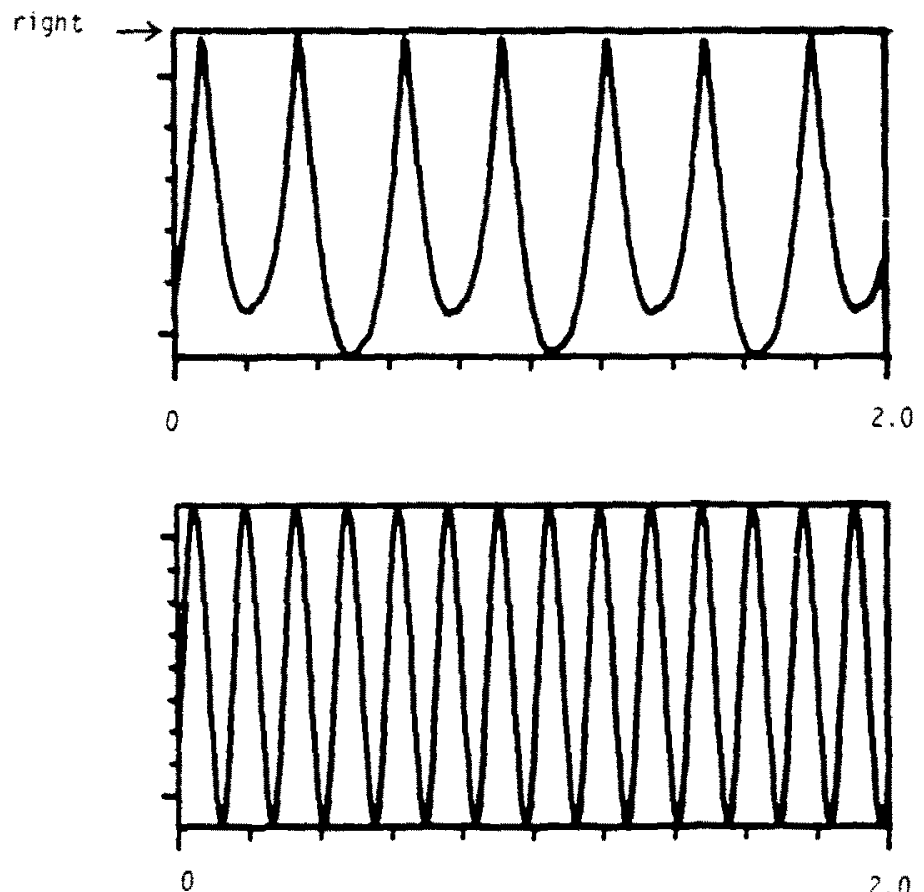

Fig. 6. Motion from period doubling bifurcation of Type I motions; inverted pendulum. Period $4 T$; letter $G$ in Fig. $5 ;(\beta, \omega)=(0.38,7.09)$.

We note that the motions associated with letters $F$ and $G$ appear at $\beta$ values lower than those predicted.

The curve denoted $\beta_{\infty}$ gives the limit curve as $n \rightarrow \infty$ for all bifurcation curves [13]. Above this curve an infinity of unstable periodic motions exist. This curve is the parameter condition above which chaos is expected to occur, although it may be only transient chaos. We notice, however, that an infinity of stable periodic motions also exist above this curve, 

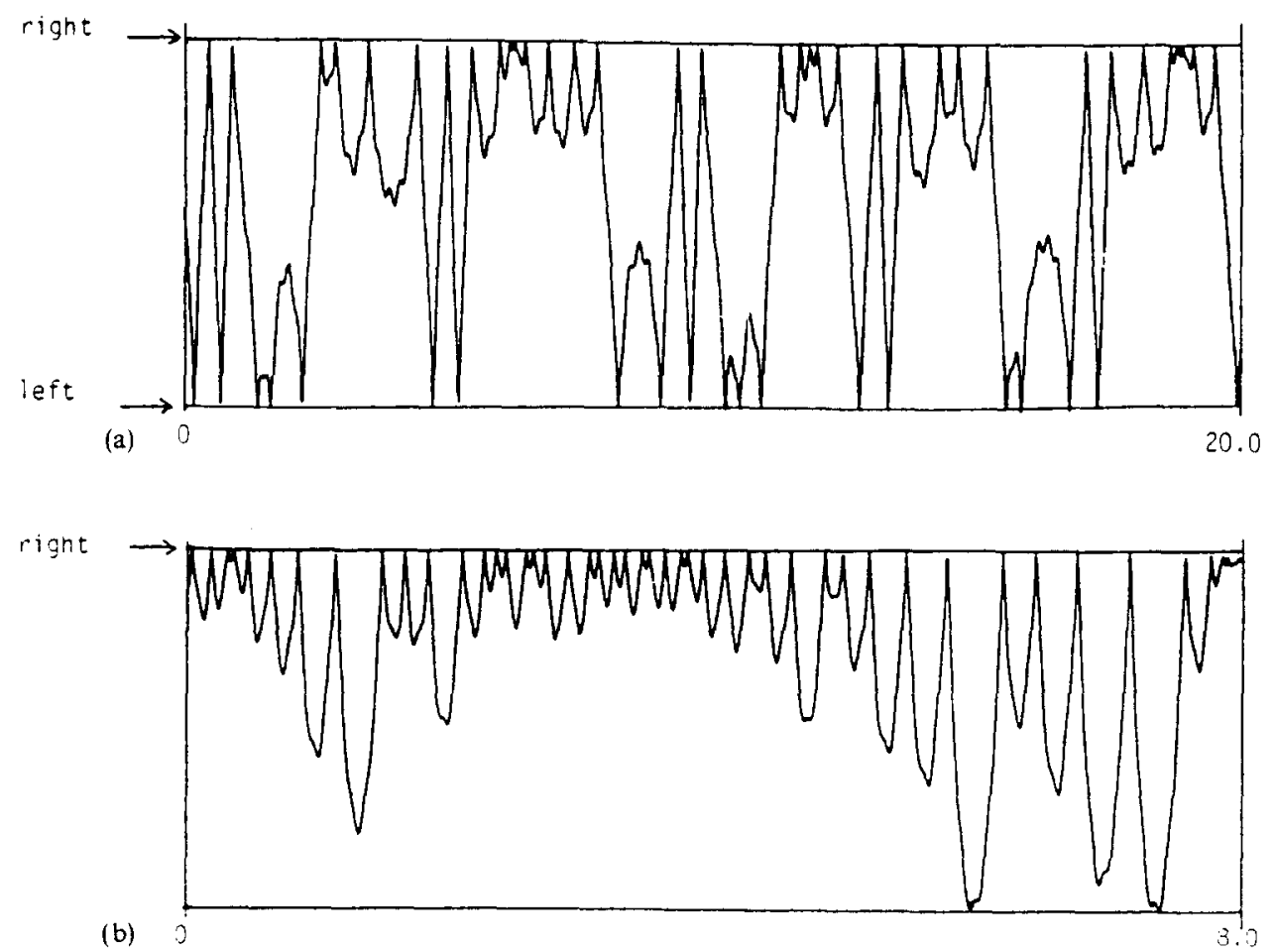

Fig. 7. Chaotic motions; inverted pendulum. (a) $(\beta, \omega)=(1.42,5.05)$. (b) $(\beta, \omega)=(1.39,10.3)$.

and thus chaos will not be the only steady-state motion possible for $\beta>\beta_{x}$. The letter $M$ corresponds to chaotic motions involving impacts at both walls; Fig. 7(a) shows an example. This is the type of chaos which can be predicted by the Melnikov analysis [13]. Chaotic motions involving impacts at only one wall were also observed; an example is shown in Fig. 7(b). These chaotic attractors must exist in pairs (due to symmetry) and merge in a global bifurcation at or before the Melnikov criteria.

The letter $\mathrm{H}$ corresponds to the rest motion in which the pendulum mass remains in contact with one of the barriers. A straightforward analysis predicts that such solutions exist for $\beta<1$ [13]. It should be noted that this static solution can coexist with stable subharmonics and/or chaotic motions. The rest solution was generally found to exist for $\beta$ values approximately less than 1.2; sample points are marked by $\mathrm{H}$ in Fig. 3. (The value 1.2 may be a consequence of stiction in the bearings.)

Other periodic motions, given by the letters $N, O$ and Q, are shown in Figs 8(a), (b) and (c), respectively. There were no theoretical predictions for the regions of existence and stability of these motions. It was expected that such periodic motions could occur but the analysis to determine their existence and stability regions is quite complicated. The letter $P$ corresponds to a motion of period $6 T$ with two impacts per period. The motions corresponding to the letters $\mathrm{I}, \mathrm{J}, \mathrm{K}$ and $\mathrm{L}$ are discussed with the Type II motions.

Figure 4 shows the experimentally observed Type II motions and the associated bifurcation curves. The difference in the $\beta$ scale in Fig. 4 compared with that in Fig. 3 is noteworthy. The Type II motions occur at larger excitation values than the Type I motions; this is intuitively correct as a larger excitation is required to push the inverted pendulum over the energy hill of the unstable unforced equilibrium position. We note that, just as for the Type I motions, there are $\beta_{n}^{\prime \prime}(\mathrm{SN})$ bifurcation curves corresponding to the appearance of Type II motions via saddle-node bifurcations. The secondary bifurcations are pitchforks in this case; the predicted values are labeled $\beta_{n}^{\prime \prime}(\mathrm{PF})$. For parameter values above the Type II saddle-node bifurcation curves, the associated motions exist and are stable until the corresponding pitchfork bifurcation curve is reached. At these points, the motions from the saddle-node bifurcations become unstable and two new antisymmetric periodic motions are born. 
The time traces of motions corresponding to letters I (Type II, period $T$ ) and J (Type II, period $3 T$ ) are given in Figs 9(a) and (b), respectively. These results do not show the close correspondence with the predicted saddle-node bifurcation curves as did the Type I motions, but the trends are as expected. Motions denoted by the letters $\mathrm{K}$ and $\mathrm{L}$ correspond to Type II motions of periods $5 T$ and $7 T$, respectively (not shown). Chaotic motions, again represented by the letter $M$, occurred widely throughout the region above the $\beta_{\infty}$ curve, and only a few were plotted for representation.

No clear examples of motions arising from the secondary pitchfork bifurcations were observed; there are two possible explanations for this. One is that the parameter region for some 'pitchfork motion' to exist is for a large excitation $(\beta)$ and low frequency $(\omega)$. Our
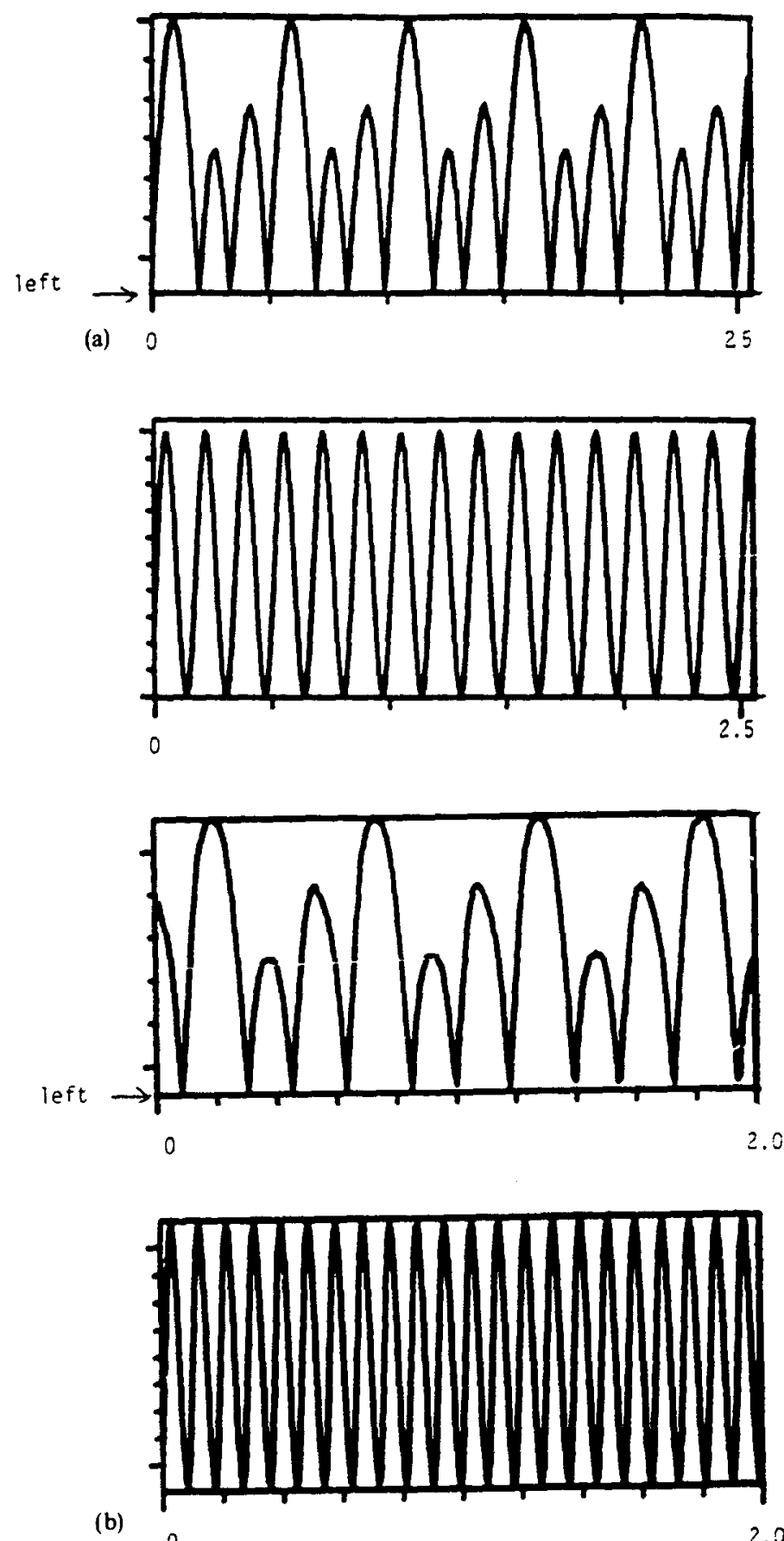

(b) 

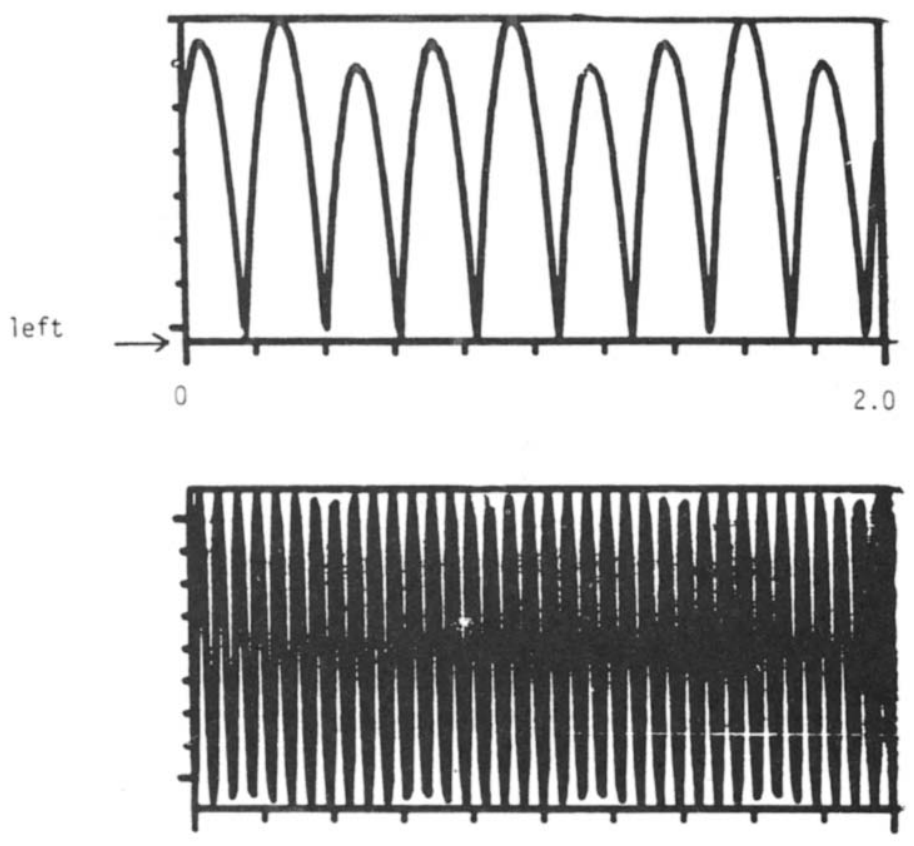

(c)

0

2.0

Fig. 8. Miscellaneous periodic motions for the inverted pendulum. (a) Period 37 ; three impacts; letter $N$ in Fig. $5 ;(\beta, \omega)=(0.45,6.08$ ). (b) Period 6T, three impacts; $O ;(0.54,11.14)$. (c) Period $12 T$, three impacts; $Q ;(0.50,18.24)$.

experimental apparatus was not able to provide excitation of this type. The second reason is that in a time trace the symmetric and unsymmetric motions look very similar; a phase portrait is often required to determine if the motion has undergone a pitchfork bifurcation.

\subsection{Normal pendulum}

Figure 10 shows the observed motions and the predicted saddle-node bifurcation curves from Shaw [7] for Type II motions of the normal pendulum system. These bifurcation curves are for the case of no free flight damping, $\alpha=0$. (The assumption of no damping is reasonable because of the low value for the actual damping and the fact that the bifurcation curves depend on the damping only to the first order.) Here steady-state linear motions and non-linear motions can coexist. Linear motions are those which do not impact the constraints, and the non-linear motions are typically either Type II, a bifurcated offspring of Type II, or chaotic. All non-linear steady-state motions impact both constraints. The $\beta_{1}(\mathrm{SN})$ curve represents parameter values above which the motions corresponding to the letter $\mathrm{X}$ are predicted to occur; this motion is of Type II, period $T$. The $\beta_{3}(\mathrm{SN})$ curve gives the parameter values above which the motions given by the letter $Y$ are expected to exist; these are period $3 T$, Type II motions. The $\beta_{5}$ (SN) curve gives the parameter values above which we expect to find the motions corresponding to the letter $Z$, Type II, period $5 T$. The curve for the $\beta_{7}(\mathrm{SN})$ was not plotted; however, stable motions arising from this bifurcation are denoted by the letter $W$.

In the normal pendulum system the secondary bifurcations are pitchfork bifurcations, just as for Type II motions in the inverted pendulum system. However, the differences between the symmetric Type II motions and their non-symmetric offspring were clearer in this case, and some motions could be classified as having been born in a pitchfork bifurcation. The motions from the pitchfork bifurcation for $n=1$ are given by the letter $\mathrm{Q}$. A time trace of such a motion is shown in Fig. 11. (The lack of symmetry in this motion can be observed by comparing the excursions between the walls, which are not symmetrical.) The conditions for the pitchfork bifurcation to occur are not plotted in Fig. 10. However, it is expected that this system responds in a similar manner to the inverted pendulum system, in the sense that there will be some parameter region for which the symmetric motion will exist and be stable, and 

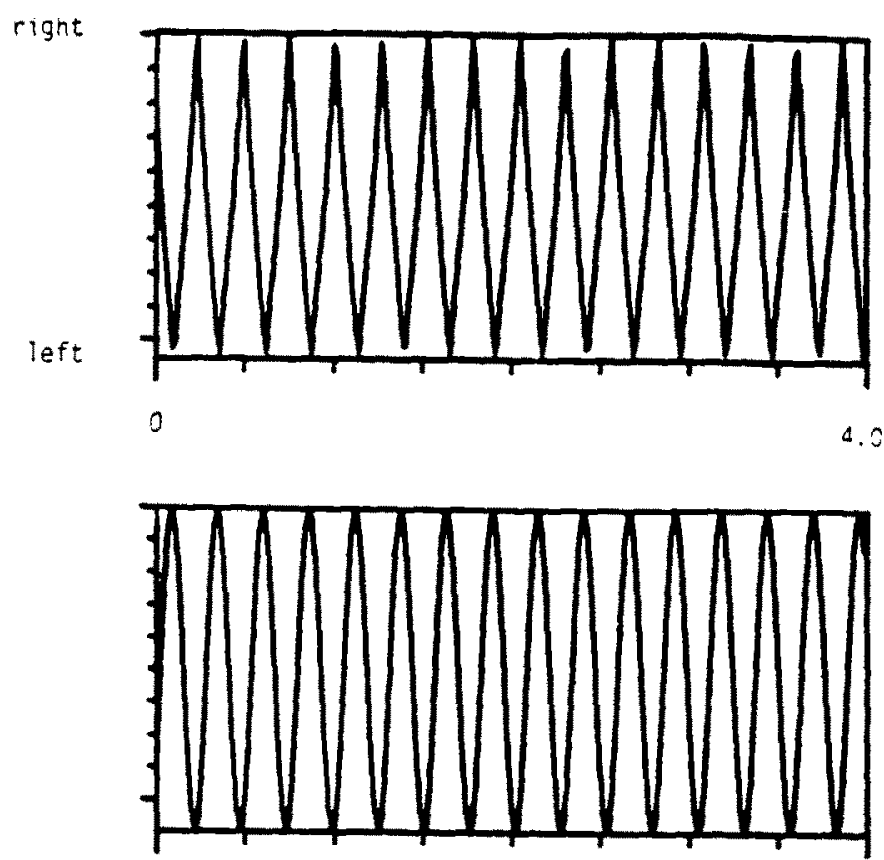

(a) 0

4.0
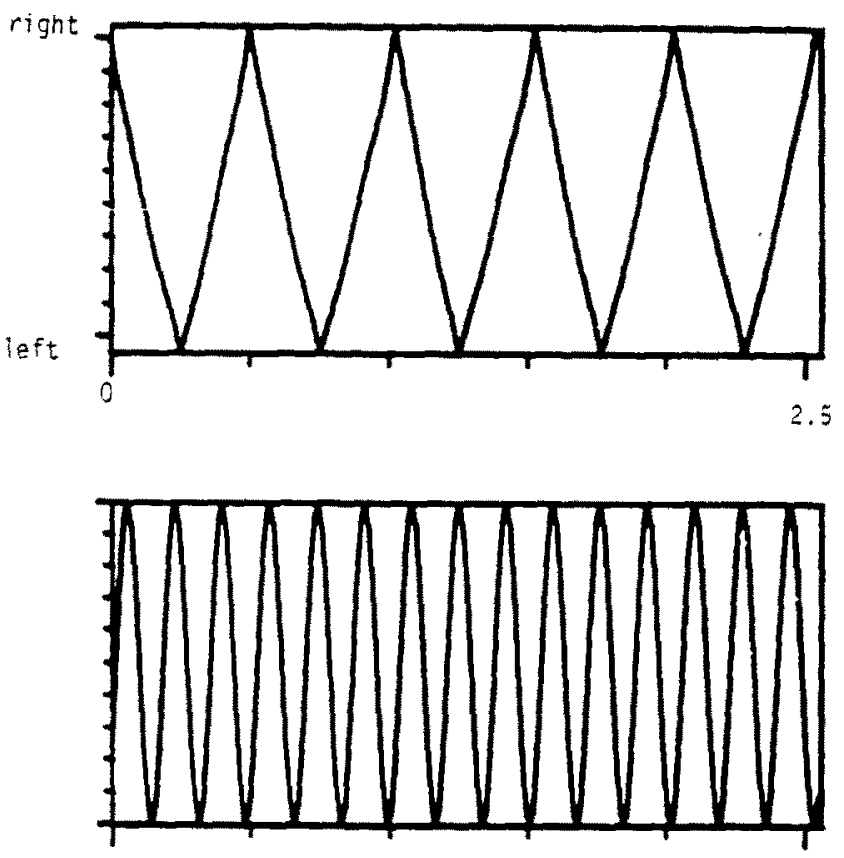

(b)

0

2.5

Fig. 9. Type II motions for the inverted pendulum. (a) Period $T$; letter I in Fig. $6 ;(\beta, \alpha)=(0.376,4.05)$. (b) Period $3 T$; letter $J$ in Fig. 6; $(0.903,5.94)$.

at $\beta_{n}(\mathrm{PF})$ a pitchfork bifurcation will occur in which that motion loses stability and the asymmetric motions will be born.

The motion denoted by the letter $R$ represents a periodic impacting motion which is not classified as Type I or II. A time trace for motion $R$ is given in Fig. 12. The letter $P$ corresponds to a periodic motion of period $6 T$, with two impacts per period. The letter $\mathrm{M}$, as for the inverted pendulum system, corresponds to the observance of a chaotic motion of the normal pendulum. The occurrence of chaos for the normal pendulum was not as widespread as for the inverted pendulum. 


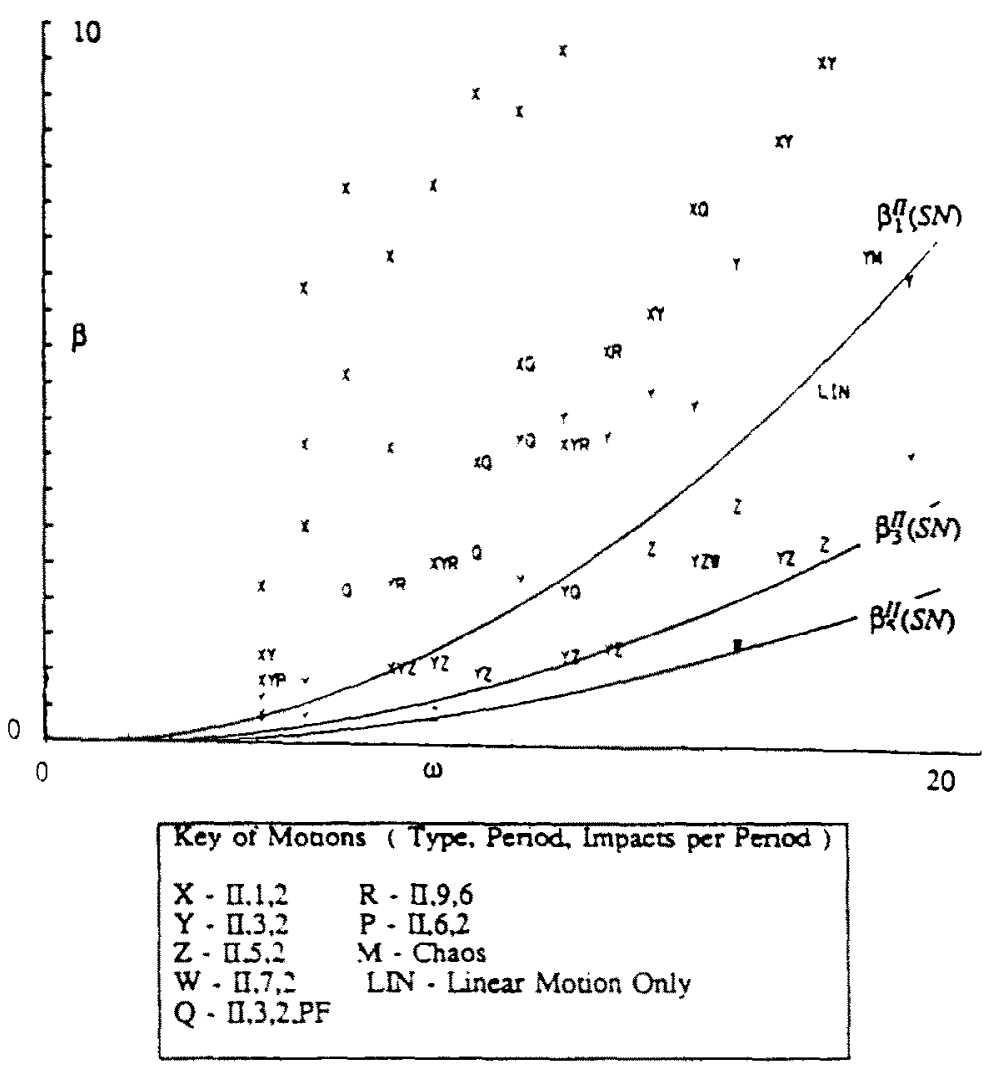

Fig. 10. Experimental results for the normal pendulum. Curves are the predicted saddle-node conditions from Shaw [7].
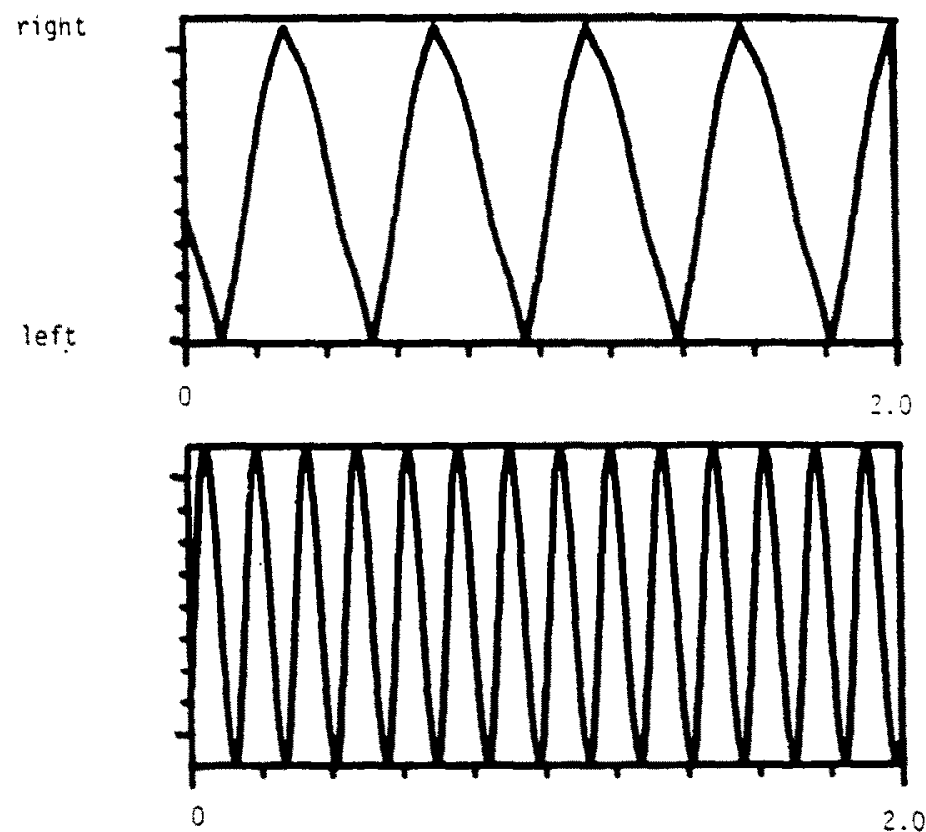

Fig. 11. Motion born in a pitchfork bifurcation from a Type ll. period $3 \mathrm{~T}$ motion, normal pendulum: letter $Q$ in Fig. 12; $(\beta, \omega)=(2.1,7.09)$.

Linear motions are not represented as they occurred at all points in the parameter space which was accessible by our equipment. (The condition for the existence of the linear steadystate is easily determined by requiring its amplitude to be less than unity, this results in the condition $\beta<\left|1-\omega^{2}\right|+\mathbf{O}(\alpha)$, which is satisfied by all parameter values examined in the experiment.) 

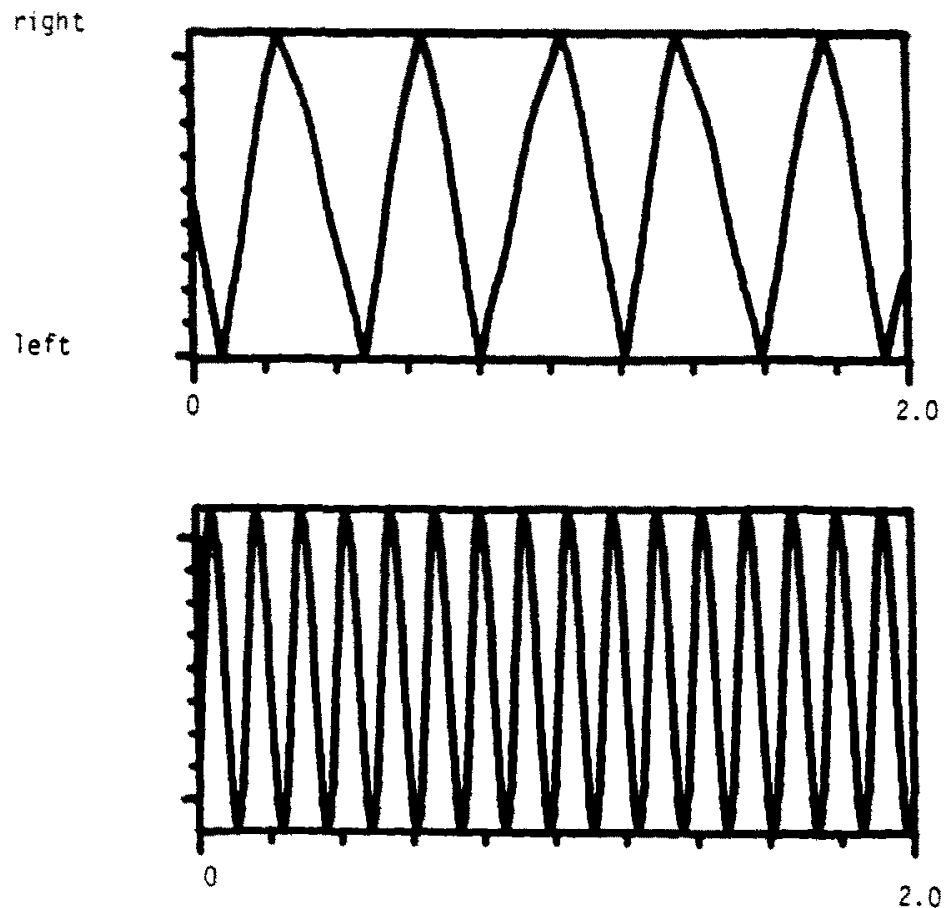

Fig. 12. Period 9T, six impacts per period motion, normal pendulum; letter $R$ in Fig. 12; $(\beta, \omega)$ $=(2.18,8.1)$.

This experiment clearly demonstrates how a system with clearances may have very complicated dynamics in a operating region where the linear steady-state response exists.

\section{DISCUSSION}

The experimental results presented in this paper compared well with those predicted by the theoretical models used for the normal and the inverted pendulum systems. The discrepancies from the predicted values arose from the assumption made in the model of the system. The assumptions of linear viscous friction in the bearing, single degree of freedom, and instantaneous impacts based on a constant coefficient of restitution are all useful approximations of the physical system but are not full descriptions of the reality of the situation. In particular, the simple impact rule becomes less valid as the impact velocity is increased. This may explain the fact that predictions for the lower $\beta$ values were reasonably accurate while for larger $\beta$ values the predictions were not as good.

Because of the piecewise linear nature of the impacting pendulum we were able to compare results from exact analyses (that is, no small parameter assumptions required) to simulations and physical experiments. In fact, this was done for a wide range of motions including linear, non-impacting motions, various types of subharmonics, and chaos.

Acknowledgements-The authors are grateful to Professor Alan Haddow and the Case Center for Computer Aided Engineering at Michigan State for use of their laboratories and equipment while carrying out these experiments. This work was supported in part by grants from NSF (MSM-8613294) and DARPA.

\section{REFERENCES}

1. M. A. Veluswami and F. R. E. Crossley, Multiple impacts of a ball between two plates, Part 1: some experimental observations. Trans. ASME J. Engng Ind. 97, $820-827$ (1975).

2. M. A. Veluswami, F. R. E. Crossley and G. Horvey, Multiple impacts of a ball between two plates, Part 2 : mathematical modelling. Trans. ASME J. Engng Ind. 97, 828-835 (1975).

3. S. F. Masri, Theory of the dynamic vibration neutralizer with motion limiting stops. Trans. ASME J. appl. Mech. 563-568 (1972).

4. S. F. Masri and T. K. Caughey, On the stability of the impact damper. Trans. ASME J.appl. Mech. 33, 586-592 (1966).

5. H. G. Davies, Random vibration of a beam impacting stops. J. Sound Vibrat. 68(4), 479-487 (1980). 
6. T. Watanabe, Steady impact vibrations of continuous elements (case of colliding once in a hall cycle). Bull. JSME 24 (1981).

7. S. W. Shaw, The dynamics of a harmonically excited system having rigid amplitude constraints, Part 1: subharmonic motions and local bifurcations. Trans. ASME J. appl. Mech. 32, 453-458 (1985).

8. S. W. Shaw, The dynamics of a harmonically excited system having rigid amplitude constraints, Part 2: chaotic motions and global bifurcations. Trans. ASME J. appl. Mech. 52. 459-464 (1985).

9. S. W. Shaw and P. J. Holmes, A periodically forced piecewise linear oscillator. J. Sound Vibrat. 90(1), 129-155 (1983).

10. P. J. Holmes. The dynamics of repeated impacts with a sinusoidally vibrating table. J. Sound Vibrat. 84,173-189 (1982).

11. J. M. T. Thompson, Complex dynamics of compliant off-shore structures. Proc. R. Soc. Lond. A, 387, 407-427 (1983).

12. G. S. Whitson, The vibro-impact response of a harmonically excited and preloaded one-dimensional linear oscillator. J. Sound Vibrat. 115(2), 303-314 (1987)

13. S. W. Shaw and R. H. Rand. The transition to chaos in a simple mechanical system. Int. J. Non-linear Mech. 24, $41-56(1989)$.

14. M. Sharif-Bakhtiar and S. W. Shaw. The dynamic response of the centrifugal pendulum vibration absorber with motion limiting stops. $J$. Sound Vibrat. 126(2), 221-235 (1988).

15. F. C. Moon and P. J. Holmes, A magnetoelastic strange attractor. J. Sound Vibrat. 65(2), 285-296 (1979).

16. F. C. Moon. J. Cusumano and P. J. Holmes, Evidence for homoclinic orbits as a precursor to chaos in a magnetic pendulum. Physica 24D, 383-390 (1987).

17. F. C. Moon and S. W. Shaw, Chaotic vibrations of a beam with non-linear boundary conditions. Int. J. Nonlinear Mech. 18(6), 465-477 (1983).

18. S. W. Shaw, Forced vibrations of a beam with one-sided amplitude constraint: theory and experiment. J. Sound Vibrat. 99(2), 199-212 (1985).

19. R. W. Leven, B. Pompe. C. Wilke and B. P. Koch, Experiments on periodic and chaotic motion of a parametrically forced pendulum. Physica 16D, 371-384 (1985).

20. B. P. Koch and R. W. Leven, Subharmonic and homoclinic bifurcations in a parametrically forced pendulum. Physica 16D, 1-13(1985).

21. M. J. Feigenbaum, Quantitative universality for a class of non-linear transformations. J. statist. Phys. 19, 25-52 (1978).

22. D. Moore, The experimental response of an impacting pendulum system. M. S. thesis, Michigan State University (1987) 